\title{
Bridge inspection quality improvement using standard inspection methods
}

\author{
Matej Kušar ${ }^{1}$ \\ DOI: https://doi.org/10.5592/CO/BSHM2017.3.7 \\ ${ }^{\text {IF }}$ aculty of Civil and Geodetic Engineering, Jamova 2, 1000 Ljubljana, Slovenia, \\ E-mails: matej.kusar@fgg.uni-lj.si;
}

\begin{abstract}
There are many approaches in structural damage detection and assessment. However, for a large number of bridges to be periodically inspected only visual inspection and non-destructive testing are suitable. European countries use different regular bridge inspection protocols resulting in divergent data quality. To improve it in countries with underperforming bridge inspection methodologies, guidelines need to be defined that will help develop appropriate visual inspection protocols. In order to accurately determine the risk of intense or concealed damage non-destructive tests have to be employed in addition. These tests should be time and cost efficient, and above all, complementary with visual inspection.
\end{abstract}

Keywords: bridge inspection, damage detection, visual inspection, non-destructive testing

\section{Introduction}

The bridge inspection process is critical to ensuring the safety of bridges, identifying repair and maintenance needs and determining appropriate allocation of funds. As a result, the quality of the data produced during the inspection process is extremely important (Washer \& Chang, 2009). The inspection process is the foundation of the entire bridge management system. Data accuracy has to be consistent throughout the period when inspections are conduced. Only then, it is possible to get the best possible information describing the bridge current state, performance, deterioration rate and similar, over the years of use.

To ensure quality data acquisition and processing a quality control plan has to be developed. This is one of the main objectives of COST Action TU1406. Its development is planned within Work Group 3 (WG3) and its framework has already been outlined (fig.1). It consists of several interconnected and interdependent parts, with the final goal to determine the performance value of each element of the individual bridge and each bridge within the addressed network as well as network as a whole.

As it can be seen (fig.1), one of the components in the proposed quality control plan is observation. The primary objective of this activity is to register type, extent and intensity for every damage recorded on each element of the bridge. During field investigations, every element is being examined separately and at the bridge component level (there are no complex analyses to be performed on this level), one of the most important goals to be reached is objective damage assessment. There are four main approaches in damage detection and assessment (Strauss et al., 2016):

- visual inspection,

- non-destructive testing,

- probing and

- structural health monitoring. 


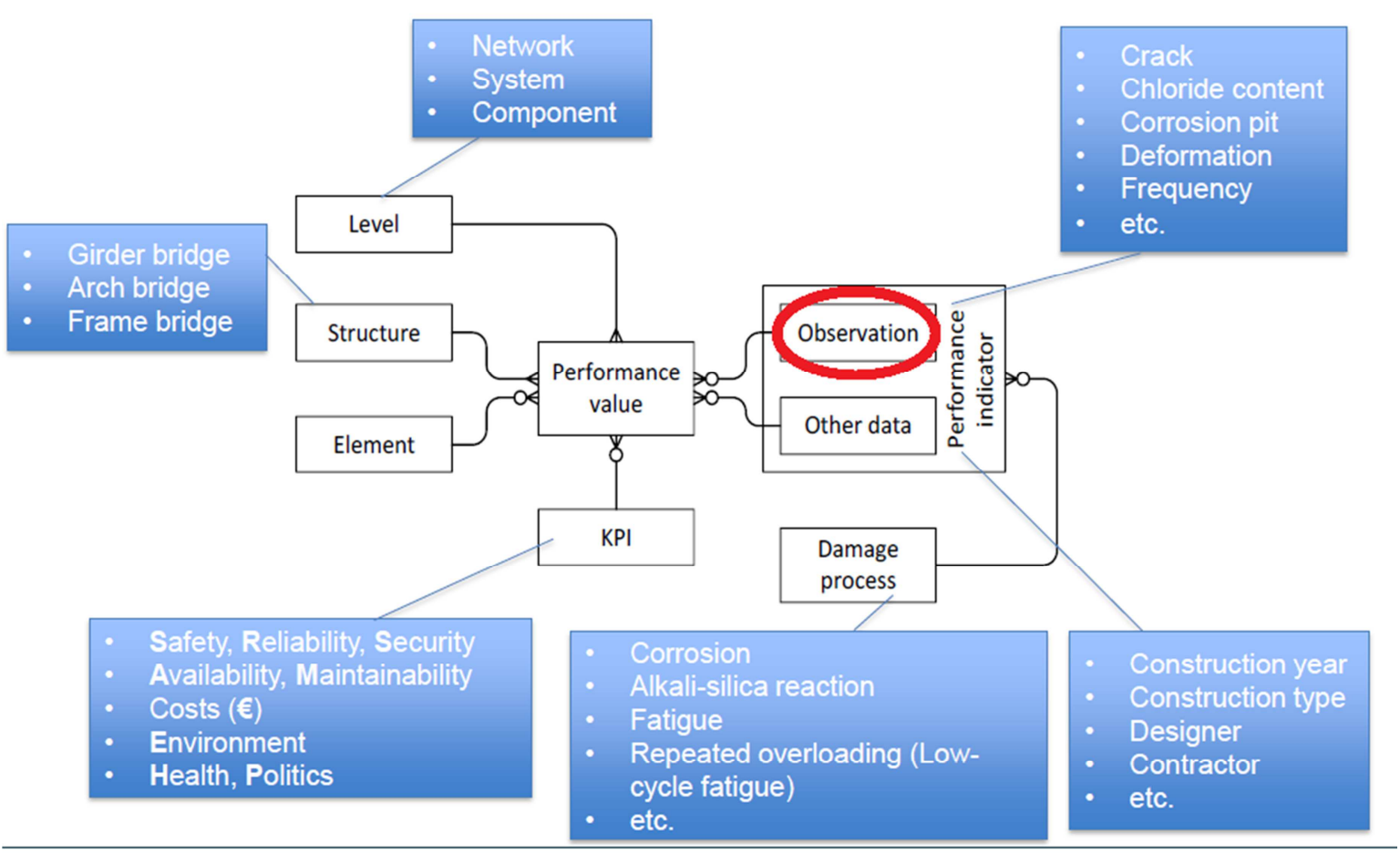

Fig. 1. Quality control plan framework (Hajdin, 2016)

Structural health monitoring (SHM) is generally performed on the bridges of utmost importance for the road network. Equipment acquisition, its maintenance, data collection and analysis require financial assets that are not affordable for large scale use. Therefore, SHM is in most cases used for bridges with large spans only. Probing provides the most reliable results regarding the state of the bridge and its individual components. Its biggest weakness is the fact that its implementation causes a certain damage to the construction. In most cases, it is performed when remediation or reconstruction of a specific bridge is already envisaged, however more accurate information on the state of the bridge components is still needed.

The use of SHM and probing is therefore not suitable for large-scale periodical damage detection and assessment. Although somewhat less reliable, for long-term data acquisition regarding the bridge state and its changes over time, two types of data collection techniques remain available: visual inspection and nondestructive testing (NDT). Both approaches have advantages and disadvantages from the viewpoint of data acquisition, reliability, work pace, required equipment etc. Most importantly, visual inspection disadvantages can be eliminated, to a large extent, with the complementary use of NDT. The use of both methods does not guarantee the quality of acquired data by itself. Appropriate quality plan and working methods have to be developed for this purpose. One of the WG3 tasks in COST Action TU1406 is to define the most appropriate use of resources available (i.e. human and equipment) for bridge inspection practice. Challenges related to this task fulfillment are addressed in the following sections.

\section{Visual inspection}

The majority of the existing bridge maintenance systems in the past were based primarily on information obtained through visual inspections (Gattulli \& Chiaramonte, 2005). Although documented past (Phares et al., 2004) and ongoing experience (Kušar, 2014) reveals that this type of inspection is often unreliable, it will remain the main aid for collecting data due to its simplicity and cost effectiveness (Tenžera et al., 2012). This point of view was accepted as an undisputed fact in WG3 meetings of COST Action TU1406 in April 2016 (Belgrade, Serbia) and October 2016 (Delft, Netherlands).

Data reliability can always be improved in two basic ways: by improvement of existing protocol of visual inspection or by use of additional methods of examination. The countries participating in the COST Action TU1406 have different visual inspection protocols. They vary greatly in manner of implementation and consequently extent and quality of the collected data. In order to develop guidelines for visual inspections, with 

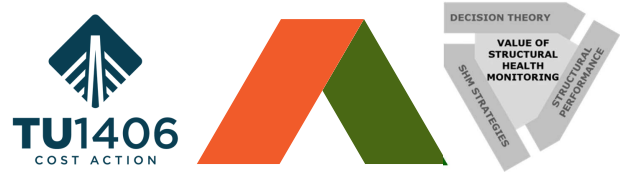

The Value of Structural Health Monitoring for the reliable Bridge Management

the aim to uniform (standardize) them as much as possible at a European level, a survey of best protocols has to be performed and subsequently, within the WG3, the issues listed in Table 1 along with some other ones need be defined.

Table 1. Some of the issues regarding visual inspections to be tackled

Frequency of inspection

Should it be fixed or dependent upon the bridge state/importance?

Inspector qualification and experience

What is the minimum formal education, when is an inspector considered experienced, do higher risk bridges need to be examined by experienced inspectors only?

Data input

Should report be completed on field or in office?

$\underline{\text { Inspectors rotations }}$

Should inspections on the selected bridge be performed always by the same inspector or should inspectors' rotation be mandatory?

Office review

Which parts of inspection reports always need review, e.g. critical findings, recommended actions, whole reports?

\section{Field review}

What percentage of bridges investigated should be reviewed? How do we select the bridges to be reviewed? By random sampling, critical bridges only, do all inspectors need to be reviewed?

Refresh training

Training extent (theoretical, practical), frequency?

\section{Performance indicators}

Should some performance indicators (e.g. scour, settlement) be given more emphasis during inspection?

There are numerus inspection manuals, presentations and inspection practices available from members of TU1406 as well as on the internet. Additionally, the majority of members have experience on the subject, therefore the only challenge is the unification of members' regarding each issue under consideration as possible methods of implementation have already been addressed in the past.

\section{Non-destructive testing}

As long as bridges exhibit no significant damage, deformations or other irregularities, visual inspections are sufficient for determining slow continuous processes of degradation. Problems occur when it becomes necessary to accurately determine the risk of intense or concealed damage (Kušar \& Cmok, 2016). Although NDT is not regularly integrated in regular bridge inspection processes, their application brings valuable additional information on the current condition of the structure and should be applied when degradation processes intensify.

Certain NDTs used for detailed examination are frequently undertaken because of their simplicity (e.g. reboundhammer, cover meter) while other methods are sophisticated and applied for special investigations or scientific use only. Reliable, relatively simple and if possible inexpensive NDT should be used for routine inspection practice. Advanced methods are not suitable for large-scale implementation, since they are in most cases expensive. An overview of selected NDT is already available in literature (e.g. Sousa et al., 2009). Additionally, a survey of over 30 methods is currently in progress (Table 2). The aim is to identify methods appropriate for use 
from the viewpoint of time consumption, cost efficiency and reliability of results. They must also be able to cover the shortcomings of visual inspections.

Table 2. NDT methods survey

\begin{tabular}{lccc}
\hline \hline & 1. & NDT for measurement of: cracks, leaking, mechanical damage, scaling, segregation... \\
\hline \hline Image Pro Plus (IPP) & Acoustic emission (AE) & Impact echo & Infrared thermography \\
\hline Impulse response & Radiography & Petrography & Lamb wave Theory \\
\hline \hline & 2. & NDT for measurement of: compressive strength, surface, hardness, adhesion \\
\hline \hline Rebound hammer & Ultrasonic pulse velocity (UPV) & CAPO test & Probe penetration \\
\hline Micro-coring & Pull-of test & \\
\hline \hline
\end{tabular}

\begin{tabular}{|c|c|c|c|}
\hline Quantab test & Potentiometric titration & Fast chloride test & \\
\hline \multicolumn{4}{|c|}{ 4. $\quad$ NDT for measurement of: corrosion } \\
\hline $\begin{array}{l}\text { Galvanostatic pulse } \\
\text { method }\end{array}$ & $\begin{array}{l}\text { X-ray diffraction and atomic } \\
\text { absorption }\end{array}$ & $\begin{array}{l}\text { Electrical potential } \\
\text { measurement }\end{array}$ & $\begin{array}{l}\text { Time domain reflectometry } \\
\text { (TDR) }\end{array}$ \\
\hline Ultrasonic waves & Linear polarization resistance & & \\
\hline
\end{tabular}

5. NDT for measurement of: carbonation (concrete $\mathrm{pH})$

\begin{tabular}{ll}
\hline \hline $\begin{array}{l}\text { Phenolphthalein } \\
\text { indicator test }\end{array}$ & Rainbow indicator \\
\hline \hline
\end{tabular}

6. NDT for measurement of: internal damage and defects, delamination

\begin{tabular}{|c|c|c|c|}
\hline $\begin{array}{l}\text { Vibration based } \\
\text { damage identification }\end{array}$ & Seismic refraction method & $\begin{array}{c}\text { Ultrasonic longitudinal } \\
\text { waves }\end{array}$ & $\begin{array}{l}\text { Ultrasonic continuous } \\
\text { spread spectrum signal }\end{array}$ \\
\hline \multicolumn{4}{|c|}{ 7. NDT for measurement of: internal damage and defects, delamination } \\
\hline $\begin{array}{l}\text { Water permeability } \\
\text { test }\end{array}$ & $\begin{array}{c}\text { Initial surface absorption test } \\
\text { (ISAT) }\end{array}$ & $\begin{array}{c}\text { Covercrete absorption test } \\
\text { (CAT) }\end{array}$ & \\
\hline
\end{tabular}

For each test presented in Table 2 the following content is to be addressed: performance indicators assessed, method application, its advantages and disadvantages, possible other issues. The main goal is to assess compatibility and complementarity of the discussed NDT with visual inspection.

\section{Conclusions}

In Europe, the state of bridges with largest spans are in most cases controlled by structural health monitoring systems, while the damage state of vast majority of bridges is determined by conducting periodical regular inspections only. Detecting damage during these inspections will remain in the domain of visual inspection, however evaluating its extent and intensity is most likely to be shared with selected NDTs.

Although somewhat contradictory to the above conclusions, it should be noted that a large number of bridges to be periodically inspected, in combination with limited financial resources, demands only reasonable and not best possible effort for quality data acquisition. The latter will stay in the domain of detailed bridge inspection. Therefore, selection of the best suitable protocol for visual bridge inspection could be extremely demanding. Use of NDTs, on the other hand, has to be thoroughly examined in order to select the methods that are most suitable, i.e. time and cost efficient, reliable and complementary with visual inspection. 


\section{References}

Vincenzo Gattulli, Leonardo Chiaramonte. Condition Assessment by visual inspection for a Bridge Management System, Computer-Aided Civil and Infrastructure Engineering, 20:95-107, 2005.

Rade Hajdin. From performance indicators to performance goals. PowerPoint presentation. COST Action TU1406 $3^{\text {rd }}$ Workshop. 20-21 October, Delft, The Netherlands, 2016.

Matej Kušar. Development of bridge management system for roads and highways. PhD thesis, Faculty of civil and geodetic engineering, University of Ljubljana, Slovenia, 2014.

Matej Kušar, Dušan Cmok. Non-destructive investigation techniques in bridge inspection. COST Action TU1406 $3^{\text {rd }}$ Workshop. 20-21 October, Delft, The Netherlands, 2016.

Brent M. Phares, Deniss D. Rolander, Benjamin A. Graybeal, Glen A. Washer, Mark Moore. Routine highway bridge inspection condition documentation accuracy and reliability. Journal of Bridge Engineering, 9(4): 403-413, 2004.

Carlos Vilela de Sousa, Joana Oliveira Almeida, Raimundo Moreno Delgado. Bridge management system as an instrument of risk mitigation. $3^{\text {rd }}$ International conference on integrity, reliability and failure. Paper ref. S2507_P0378. Porto, Portugal, 20-24 July, 2009.

Alfred Straus, Ana Mandič Ivanković, Jose C. Matos, Joan R. Casas. Performance indicators for roadway bridges of Cost Action TU1406. WG1 Technical report. 2016.

Danijel Tenžera,, Goran Puž, Jure Radić. Visual inspection in evaluation of bridge condition. Građevinar, 64: 717-726, 2012.

Glen A. Washer, Alec C. Chang. Guideline for implementing quality control and quality assurance for bridge inspection. National Cooperative Highway Research Program, Project 20-07, Task 252. 2009. 